\title{
Interactive comment on "Seismic anisotropy inferred from direct S-waves derived splitting measurements and its geodynamic implications beneath southeastern Tibetan Plateau" by Ashwani Kant Tiwari et al.
}

\section{Anonymous Referee \#2}

Received and published: 7 November 2016

\section{Summary}

This manuscript describes about 5,000 measurements (500 averages) of shear wave splitting using $S$ waves at 46 seismic stations in southeastern Tibet. The authors correct for receiver-side splitting using the reference station technique, and account for source-side anisotropy somehow as part of this process They assume that SKS shear wave splitting can be used for the receiver correction. They find generally good agreement with previous, SKS-based results, save a few stations where SKS was null. They interpret their observations in light of previous work studying the tectonics of the region, 
suggesting that deformation in both the lithosphere and, separately, the asthenosphere account for their observations.

Overall, I think the observations are well made and the interpretations are fairly reasonable, so subject to some revision it should be suitable for publication in Solid Earth. I have confidence in the observations, and the use of a novel method is interesting. However, it should be noted that using such short distances to measure splitting in $S$ may be problematic and the authors must check this. The paper is less strong in terms of dynamical implications, but I do not feel these are the focus of the study. There are quite a lot of grammatically incorrect sentences and the writing needs to be corrected before publication, though only rarely is the sense lost.

\section{Major points}

1. The whole analysis relies on SKS splitting measurements being a useful way to correct receiver-side splitting in $S$ waves. This is probably true at long distances, but probably less and less accurate as epicentral distances get shorter, when the ray path and incidences angles are quite different. The authors should, I suggest, show that there is no systematic variation in the $S$ splitting parameters with distance. It would also be a good idea to test that excluding shorter-distance observations (say, less than $50^{\circ}$ ) does not change their average splitting parameters.

2. A related point: do the SKS measurements that are used as corrections show any 'complex' behaviour? That is, variation of splitting parameters with backazimuth? The cited reference (Sol et al., Geology, 2007) does not list individual splitting measurements so it is impossible to know-but given the complexity of the region and the apparent 'null' stations (as the authors argue), it seems very likely that multiple anisotropic layers might be needed to explain observations. To that end, what is the uncertainty in these $S$ wave splitting measurements due to (a) the variation in SKS splitting with 
backazimuth, and (b) the fact that SKS and S polarisations may not be the same?

3. The authors use epicentral distances down to $30^{\circ}$. At this distance, it's likely that shear-coupled $P$ phases will interfere with the $S$ arrival and may induce apparent splitting (e.g., Wookey \& Kendall, JGR, 2004). Again, checking for distance-dependence in splitting parameters may help identify this, and applying wavefield decomposition (e.g., Kennett, GJI, 1991) can be done to se if this makes a difference. Either way, it is a concern which should be addressed, because I see that very many events have been used which are very close (in terms of teleseismic splitting).

4. How deep are the events used here? Shallow earthquakes can lead to interfering depth phases which induce apparent splitting. It would also be useful to know, as a test of the efficacy of the removal of the source term, what the source side splitting measurements are which are retrieved using this method. This information is also useful (Lynner \& Long, JGR, 2014; Nowacki et al., G3, 2015) for other purposes. One would expect, as seen in these papers, a decrease in the source-side splitting with depth until the transition zone, where it seems to level off, but not a dramatic change in the amount of splitting in the top $\sim 100 \mathrm{~km}$. The presence of this might indicate contamination with depth phases.

5. Despite reading both the manuscript and Eken \& Tilmann (BSSA, 2014), it took me some time to grasp the method, and I think it could be a bit clearer. My current grasp of it is the following: (1) An SKS measurement previously made at the reference station is removed from the reference station traces by application of the appropriate inverse splitting operator. (2) The best-fitting fast orientation and delay time at the target station is recovered which makes the uncorrected target and corrected reference signals match; this is done by grid search over all splitting operators. This corresponds to finding the splitting operator which restores the target trace to the reference trace with only the source-side splitting imposed, thus giving the receiver-side splitting. I think many readers would appreciate a slightly fuller exposition of how this all works to remove the source-side splitting. I think the authors do a good job explaining the

Printer-friendly version

Discussion paper
Interactive

comment 
assumptions, however.

6. The authors report splitting measurements at each station-these are I think respectively the circular and linear means of the fast orientation and delay time for the set of reference stations used at that target station, but which reference stations went into each target station? All those within a $300 \mathrm{~km}$ radius? I can't find this information, which is necessary to reproduce the study. It should probably be added to Table 1. (See also my later point regarding supplementary material.)

7. The authors should also supply a list of the earthquakes used alongside Figure 2.

8. Have the authors implemented the updated F-test as described by Walsh et al. (JGR, 2013)? Or the one originally used by Silver \& Chan (JGR, 1991)? The former has pointed out some errors in the original formulation which leads to underestimates of uncertainties.

\section{Less major points}

p. 1, I. 2. '...splitting of the direct S-waves.' This is the first of many misplaced or absent articles, both definite and indefinite, throughout the manuscript, making some of it incorrect grammatically. I haven't corrected or noted all of these. These issues should be fixed before publication.

p. 2, I. 22. Particle motion does not have to be elliptical if splitting delay times are large compared to the dominant frequency. It will then be cruciform.

p. 2, I. 27. 'vertical transverse models ... with [a] horizontal axis of the [sic] symmetry.' 'Vertical transverse' isotropy only applies when the axis of symmetry is vertical. The authors mean 'horizontal transverse isotropy' $(\mathrm{HTI})$, which is hexagonal symmetry with a horizontal symmetry axis. 
p. 4, I. 14. '... show the characteristic of the [sic] splitting with clear energy on the transverse component ...' S waves are not polarised parallel to backazimuth, so there will in general be energy on both radial and transverse in isotropic models, depending on the source polarisation.

p. 4, I. 29. The authors use an upright triangle symbol for the time shift, but I think they mean to use a capital Greek delta $(\Delta)$. This is true throughout.

Interactive

comment

Table 1. Please supply this information electronically (i.e., in a plain-text ASCIl file or spreadsheet). It would be a great help to future researchers who can then avoid trying to get the PDF file's information into a more useful form.

Figure 1. It would be helpful to include station names. Either the authors could use a subfigure, or simply enlarge the region around the stations.

Figure 3. A scale bar for delay times is needed here too.

Figure 7. There appears to be a plotting error where the fault traces are all cut off at $98^{\circ}$ longitude. I also think that the subfigures could be larger and focussed more on the region around the stations, to make the splitting bars easier to see. They could also be thicker.

Figure 9. What is the scale for the lengths of the bars, GPS velocity arrows, and plate motion arrows?

Figure 9. What is the physical significance of the no-net rotation frame used for the absolute plate motion vectors? I do not see why this was used rather than one based on hot spot tracks, for instance.

Figure 9. Subfigure (c) is hard to see with the choice of colours. Perhaps choosing something other than orange would help.

Interactive comment on Solid Earth Discuss., doi:10.5194/se-2016-134, 2016. 